\title{
Acute systemic lupus erythematosus on the acute medical take: are we missing anything?
}

\author{
Elizabeth S Perry, Simon V Davies and Catherine Laversuch
}

\section{Case presentation}

\section{History and examination}

A 42-year-old man with a background of systemic lupus erythematosus (SLE) presented acutely to the medical admissions unit (MAU) with pleuritic chest pain, shortness of breath and arthralgia. He was receiving no regular medications and denied any other medical problems, any cough, recent flights, calf pain or immobility. He described the chest pain as sudden onset, right sided and exacerbated by deep inspiration. He rated the pain as $10 / 10$ severity and required opiate analgesia on admission to the MAU. On clinical examination the pain was increased by palpation at the right inferior anterior costal margin. The remainder of systems examinations were unremarkable with no rash, no synovitis and no audible pleural or pericardial rub.

\section{Baseline investigations}

On admission he was apyrexial, he had a good blood pressure of $132 / 78$ and normal oxygen saturations at $98 \%$ on air. Heart rate and respiratory rate were raised at 88 /minute and 24/minute respectively. He had an entirely normal arterial blood gas (ABG) on room air with a $\mathrm{PO}_{2}$ of $12.1 \mathrm{KPa}$. His chest X-ray (CXR) was within normal limits and his electrocardiogram (ECG) is illustrated in Fig 1.

\section{What is the differential diagnosis and the most likely diagnosis?}

\section{Acute SLE with serositis}

This presentation is highly suggestive of an acute SLE flare resulting in pleuritic chest pain secondary to serositis. This is supported by raised inflammatory markers (Table 1) and saddle shaped ST elevation on ECG (Fig 1) consistent with pericarditis.

\section{Pulmonary embolus}

Patients with SLE are at increased risk of thrombosis and care must be taken not to miss a pulmonary embolus. Despite the presence of a normal $\mathrm{PO}_{2}$ and a Wells Score of zero ${ }^{1}$ suggesting a low pre-test probability, pulmonary embolus must be considered.

Elizabeth S Perry, specialty registrar, rheumatology and general internal medicine; Simon V Davies, consultant haematologist; Catherine Laversuch, consultant rheumatologist

\section{Lower respiratory tract infection}

This is a common cause of pleuritic chest pain, but very unlikely here. To be able to attribute this degree of pleuritic pain to infection you would expect clinical findings consistent with infection, eg productive cough, coarse crepitations, bronchial breathing, pyrexia or consolidation on CXR.

\section{What is the initial management?}

Paracetamol, naproxen and codeine phosphate were used for analgesia but the pain persisted requiring morphine before it was controlled. A D-dimer was sent urgently which was raised (Table 1).

\section{Table 1. Blood results.}

Previous results

Antinuclear antibody positive $>1 / 320$ (homogenous pattern)

Extractable nuclear antigen antibodies negative

Antiphospholipid antibodies:

- anticardiolipin antibody of IgG and/or IgM isotype (measured by Elisa) - negative

- lupus anticoagulant test (measured according to international guidelines) - negative

Results on admission

Baseline bloods (reference ranges)

WCC $12.8310^{9} /$

ESR $35 \mathrm{~mm} / \mathrm{h}(1-7)$

$\mathrm{Hb} 12.1 \mathrm{~g} / \mathrm{dl}$ CRP $54 \mathrm{mg} / \mathrm{l}(<10)$

Platelets $173 \times 10^{9} / /$ D-dimer 3.2 FEU/ml (elevated)

Neutrophils $9.88 \times 10^{9} / /$ Urea and electrolytes Normal range

Lymphocytes $0.81 \times 10^{9} / \mathrm{I}$ Liver function tests Normal range Clotting screen Normal range

Serology

dsDNA $>300(<30)$

C3 $0.55 \mathrm{~g} / \mathrm{l}(0.55-1.2)$

$\mathrm{C} 4<0.1 \mathrm{~g} / \mathrm{l}(0.15-0.50)$

Antiphospholipid antibodies:

- anticardiolipin antibody of IgG and/or IgM isotype (measured by Elisa) - negative

- lupus anticoagulant test (measured according to international guidelines) - positive

$\mathrm{CRP}=\mathrm{C}$-reative protein; $\mathrm{ESR}=$ erythrocyte sedimentation rate; $\mathrm{Hb}=$ haemoglobin; IgG = immunoglobulin $\mathrm{G}$; IgM = immunoglobulin $\mathrm{M}$; $\mathrm{WCC}=$ white cell count; FEU $=$ fibrinogen equivalent units. 


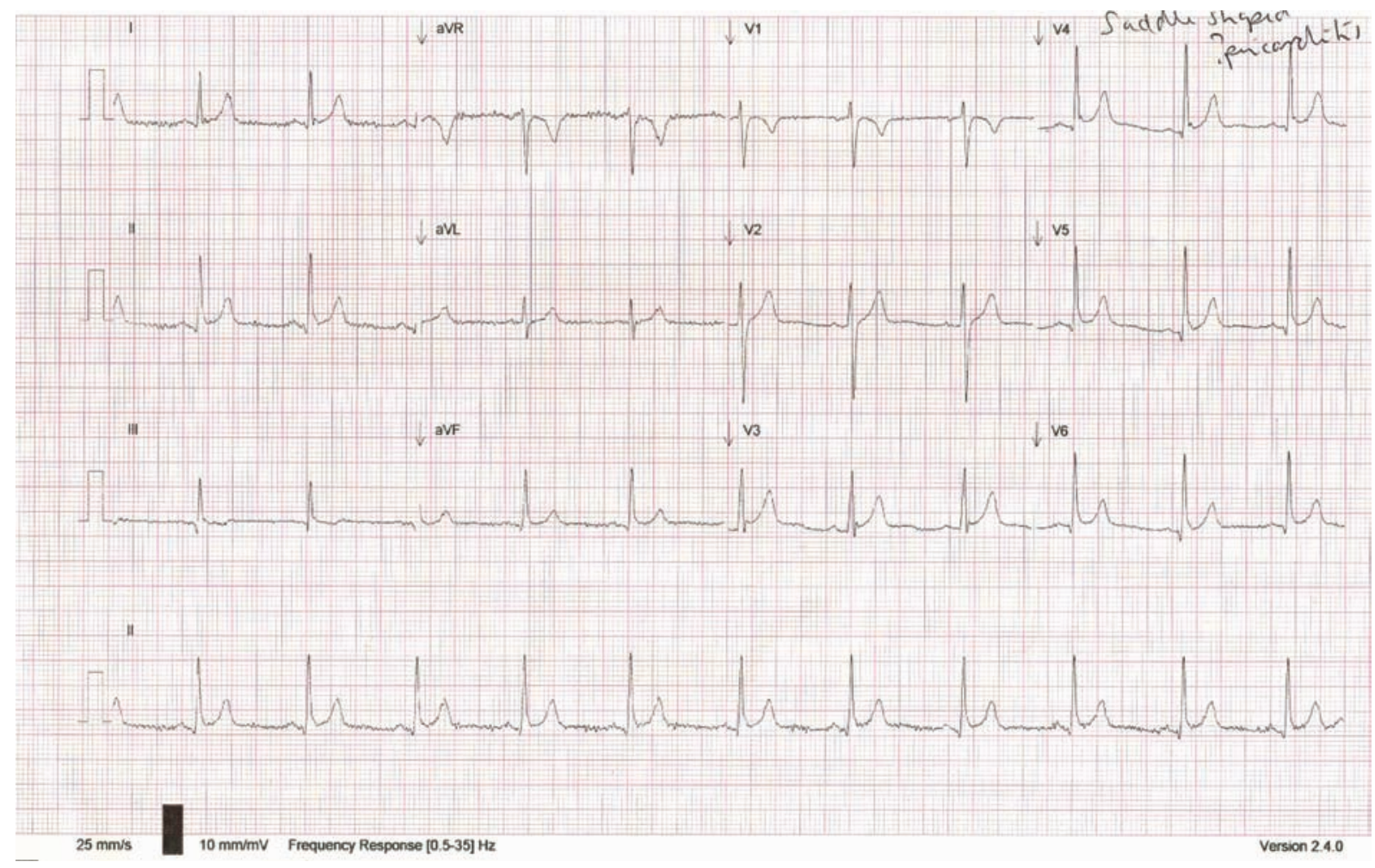

Fig 1. The admission 12-lead electrocardiogram.

The admitting team started a treatment dose of low molecular weight heparin (LMWH) for pulmonary embolism and commenced a course of corticosteroids (initially $30 \mathrm{mg}$ prednisolone once daily) to treat the acute SLE flare.

Baseline blood tests were requested (Table 1). Renal function and urinalysis were within normal limits indicating no evidence of lupus nephritis.

\section{Case progression}

\section{Computed tomography pulmonary angiogram}

Computed tomography pulmonary angiogram identified large bilateral pulmonary emboli (PE) in the upper and lower lobe arteries. Treatment dose LMWH was continued until a therapeutic international normalised ratio (range 2-3 units) was established with warfarin.

\section{Further investigations}

Double stranded DNA levels were high and complement levels were low consistent with acute SLE. Four months earlier, tests for antiphospholipid (aPL) antibodies were negative. Repeat testing on admission revealed a positive lupus anticoagulant (LA) (Table 1.)

\section{Does this patient have secondary antiphospholipid syndrome?}

From the information presented this patient does not meet the diagnostic criteria for aPL syndrome (APLS) (Table 2). ${ }^{2}$
Transient aPL positivity is common in the general population and documentation of persistence with readings greater than 12 weeks apart is crucial. Furthermore, measurement of lupus anticoagulant is unreliable in patients if acquired while on warfarin, unfractionated heparin or LMWH. It is therefore important that aPL are measured on diagnosis of SLE and not simply in reaction to an acute event.

Although a test for anti-ß-2-glycoprotein-1 is included in diagnostic criteria for APLS it is incorporated in our existing lupus anticoagulant and anticardiolipin antibody testing and is currently not measured as a separate test in routine practice outside of research centres.

\section{Key learning points}

- Systemic lupus erythematosus (SLE) patients are at increased risk of venous thrombosis even if they do not have antiphospholipid syndrome (APLS).

- Thrombosis must be considered in all SLE patients presenting with features of disease flare.

- A normal arterial blood gas does not exclude a pulmonary embolus.

- Persistent positivity for antiphospholipid antibodies with readings $>12$ weeks apart is required for a diagnosis of APLS.

- Measurement of lupus anticoagulant cannot be done on warfarin and may be unreliable in patients if acquired during an acute event or while on heparin.

- All patients diagnosed with SLE should have antiphospholipid antibodies measured at diagnosis. 
Table 2. Revised criteria for the antiphospholipid antibody syndrome. ${ }^{2}$

Clinical criteria (one or more must be present)

1 Vascular thrombosis: One or more objectively confirmed episodes of arterial, venous or small vessel thrombosis occurring in any tissue or organ.

2 Pregnancy morbidity:

- one or more unexplained deaths of a morphologically normal fetus at or beyond the 10th week of gestation or

- one or more premature births of a morphologically normal neonate before the 34th week of gestation because of eclampsia, pre-eclampsia or placental insufficiency or

- three or more unexplained consecutive spontaneous abortions before the 10th week of gestation.

Laboratory criteria (one or more must be present on two or more occasions at least 12 weeks apart using recommended procedures)

1 Lupus anticoagulant.

2 Anticardiolipin antibody of IgG and/or IgM isotype, present in medium or high titer.

3 Anti-fl2-glycoprotein-1 antibody of IgG and/or IgM isotype present in titer greater than the 99th percentile.

At least one clinical criterion and one laboratory criterion must be present for a diagnosis of APLS

APLS = antiphospholipid syndrome; IgG = immunoglobulin $\mathrm{G}$

$\operatorname{lgM}=$ immunoglobulin $\mathrm{M}$.

\section{Case outcome}

This patient made an excellent recovery. He was discharged home after five days and is currently on warfarin. His SLE is now well controlled using azathioprine and hydroxychloroquine.

\section{Discussion}

\section{Thrombosis in SLE}

Although the risk of thrombosis in SLE patients with APLS is well recognised, the risk from SLE alone is not. The incidence of thrombosis in individuals with SLE but without APLS is in the region of sixfold greater than that of the general population. ${ }^{3}$ In addition, the incidence of hereditary thrombophilic defects is thought to be higher in the SLE population ${ }^{3}$ and coexistence of two or more inherited or acquired thrombophilic defects incurs a 20-30-fold increased risk of thrombosis. ${ }^{3}$ Risk scoring systems for PE, such as the Wells Score, ${ }^{1}$ give no mention of SLE or indeed other inflammatory conditions recognised to increase thrombotic risk. Increased awareness is required.

This case illustrates a diagnostic challenge to the clinician. Although the mechanism by which SLE increases thrombotic risk is multifactorial and is yet to be fully elucidated there is a strong association between systemic inflammation and venous thrombosis. ${ }^{4}$ Indeed, the risk of venous thrombosis in patients with SLE is highest in the first year following diagnosis ${ }^{5}$ hence correlating with a time period when SLE is typically more active. It is essential that thrombosis is considered in all SLE patients presenting with features of disease flare and especially those with pleuritic chest pain.

\section{Duration of anticoagulation}

Unlike in APLS, where guidelines recommend lifelong warfarin, ${ }^{6}$ there is currently no consensus on duration of anticoagulation in cases like this where patients with SLE develop thrombosis but without antiphospholipid antibodies (sometimes referred to as seronegative APLS ${ }^{4}$ ). Many clinicians argue that lifelong warfarin is indicated given the relapsing and remitting nature of SLE, however, an alternative is to attempt to quantify thrombotic risk to inform clinical judgement. Such a thrombotic risk assessment should include assessment of disease activity/severity as well as co-existent inherited and acquired thrombophilic defects.

\section{Future directions}

Recent studies have suggested that the D-dimer assay may be a useful marker of future thrombotic events in SLE. ${ }^{7}$ However, further research is required to elucidate the pathogenesis of thrombosis in SLE and to develop an evidence-based consensus on the best practice for primary and secondary prevention of venous thrombosis in SLE in patients who do not have APLS.

\section{References}

1 Wells PS, Anderson DR, Rodgers M et al. Derivation of a simple clinical model to categorize patients' probability of pulmonary embolism: increasing the model's utility with simpliRED D-dimer. Thromb Haemost 2000;83:416-20.

2 Pierangeli SS, Chen PP, Raschi E et al. Antiphospholipid antibodies and the antiphospholipid syndrome: pathogenic mechanisms. Semin Thromb Hemost 2008;34:236-50.

3 Brouwer JL, Biji M, Veeger NJ et al. The contribution of inherited and acquired thrombophilic defects, alone or combined with antiphospholipid antibodies, to venous and arterial thromboembolism in patients with systemic lupus erythematosus. Blood 2004;104:143-8.

4 Erkan D. Lupus and thrombosus. J Rheumatol 2006;33:1715-17.

5 Sarabi ZS, Chang E, Bobba R et al. Incidence rates of arterial and venous thrombosus after diagnosis of systemic lupus erythematosus. Arthritis Rheum 2005;53:609-12.

6 Bertsias G, Loannidis JPA, Boletis J et al. EULAR recommendations for the management of systemic lupus erythematosus. Report of a Task Force of the EULAR Standing Committeee for International Clinical Studies Including Therapeutics. Ann Rheum Dis 2008;67:195-205.

7 Wu H, Birmingham DJ, Rovin B et al. D-dimer level and the risk for thrombosis in systemic lupus erythematosus. Clin J Am Soc Nephrol 2008;3:1628-36.

Address for correspondence: Dr ES Perry, Rheumatology Department, Royal Cornwall Hospital, Truro,

Cornwall TR1 3LJ.

Email: e.perry@nhs.net 